\title{
Perspective
}

PERSPECTIVE Actualité en histoire de l'art

1 | 2006

Antiquité/Moyen Âge

\section{Sculpture romane. Approche régionale, présentation monographique}

\section{Andreas Hartmann-Virnich}

\section{(2) OpenEdition}

1 Journals

Édition électronique

URL : http://journals.openedition.org/perspective/4150

DOI : $10.4000 /$ perspective. 4150

ISSN : 2269-7721

Éditeur

Institut national d'histoire de l'art

\section{Édition imprimée}

Date de publication : 31 mars 2006

Pagination : 121-124

ISSN : 1777-7852

\section{Référence électronique}

Andreas Hartmann-Virnich, «Sculpture romane. Approche régionale, présentation monographique », Perspective [En ligne], 1 | 2006, mis en ligne le 31 mars 2018, consulté le 01 octobre 2020. URL : http:// journals.openedition.org/perspective/4150; DOI : https://doi.org/10.4000/perspective.4150 


\section{Sculpture romane. Approche régionale, présentation monographique}

\section{Andreas Hartmann-Virnich}

- Anne AUTISsiER, La sculpture romane en Bretagne. $X I^{e}$-XII ${ }^{e}$ siècles, (Art et société), Rennes, Presses universitaires de Rennes, 2005, 380 p., 348 ill. ISBN : 2-7535-0066-5 ; $26 €$.

- Évelyne Proust, La sculpture romane en Bas-Limousin. Un domaine original du grand art languedocien, Paris, Picard, 2004. 355 p., 403 fig. ; photographies de Jean-François Amelot. ISBN : 2-7084-0705-8 ; $80 €$.

- Marcello AngheBen, Les chapiteaux romans de Bourgogne: thèmes et programmes, (Culture et société médiévales), Turnhout, Brepols, 2003. 559 p., 151 photographies n. et b., 43 plans ; photographies d'Ivan Verzar. ISBN : 2-50351299-2 (br.) ; $69 €$.

- Caroline Roux, La pierre et le seuil. Portails romans en Haute-Auvergne, (Études sur le Massif central),

Clermont-Ferrand, Presses universitaires Blaise-Pascal, 2004. 378 p., 125 fig. n. et b. ISBN : 2-84516-259-6; $28 €$.

Bâtir l'étude régionale (fondement de l'approche de la diversité, de la diffusion et de l'évolution de l'art roman) sur un corpus raisonné, compenser les carences bibliographiques et dégager la trame d'une réflexion cohérente sur les œuvres et leur contexte chronologique, géographique et artistique : tel est le propos de deux ouvrages issus du Centre d'études supérieures de civilisation médiévale (CESCM) de Poitiers, dont la monographie sur Notre-Dame-la-Grande reste un des jalons majeurs des dernières années dans le domaine ${ }^{1}$. La thèse d'Anne Autissier, La sculpture romane en Bretagne, et l'étude d'Évelyne Proust, La sculpture romane en Bas-Limousin, illustrent chacune à sa manière les enjeux et difficultés de la synthèse régionale. L'ouvrage de Marcello Angheben, Les chapiteaux romans de Bourgogne, explore l'approche iconographique, un des points forts de la recherche au sein du CESCM.

Pour aborder un sujet vaste et peu traité, l'étude d'Anne Autissier privilégie la géographie et la typologie formelle du décor, entre "les chantiers majeurs adeptes du corinthien " et ceux de la mouvance géométrique. Réservée au catalogue de la fin de l'ouvrage (cinquante-huit notices, p. 249360), l'appréciation de la dimension architecturale, que l'état hétérogène des monuments rend souvent délicate, reste en marge dans cette synthèse, selon un parti pris qui subordonne la « fonction architectonique importante " du chapiteau à "sa fonction décorative certaine ". Dans la recherche de critères du classement pour une géographie et une chronologie des décors, certaines notions commel' « ins-

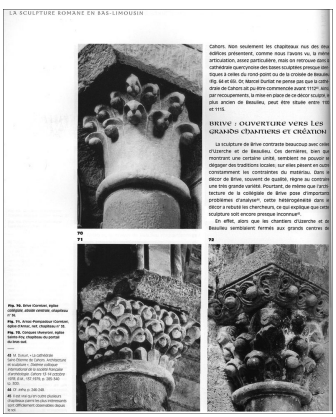

1. PROUST, p. 58. piration " ou l'«exubérance " tiennent d'une démarche intuitive, et placer l'absence des "schémas antiquisants " sous le signe du " goût " artistique et de l'«expression décorative" (p. 127 et 157) induit une enquête sur " les chantiers qui développent le géométrisme " principalement centrée sur la forme. En délaissant des questions significatives comme la relation avec le chapiteau cubique germanique ou le rapport du "répertoire géométrique savant " avec une possible stylisation à l'extrême du registre végétal, le classement proposé prend le risque de confondre l'aspect et le sens, d'écarter les avatars de la réduction extrême du schéma corinthien (fig. 104, 107) et de sousestimer les limites d'une étude comparative et diachronique des schémas les plus élémentaires au-delà de l'échelle micro-régionale (p. 155). Si ces réserves pèsent peu face à l'ampleur du travail accompli, l'enquête est à poursuivre.

L'étude d'Évelyne Proust, La sculpture romane en Bas-Limousin, se place dans une perspective similaire, ce qui se reflète autant dans la structure de l'ouvrage que dans la méthode. Le livre est assorti d'un riche dossier photographique qui met en relief la nature du travail de sculpture jusque dans la texture même de la pierre, tout en ajoutant un réel plaisir esthétique. À la lecture de ce bel ouvrage surgissent les mêmes interrogations : fautil élargir l'exposé historique jusqu'aux temps préhistoriques, au prix de carences bibliographiques inévitables ? Quant à l'approche de la sculpture architecturale en tant que telle, l'absence de l'archéologie monumentale pose la question du sens de la chronologie relative des monuments et de leur décor sculpté, bien que les vingt-quatre notices monographiques (p. 211-344), qui complètent utilement la synthèse, laissent espérer, comme dans le cas de l'ouvrage sur l'architecture romane de Bretagne, une future reprise des études archéologiques des édifices, souvent de date ancienne, et un renouvellement méthodologique. 
2. ANGHEBEN, p. 484-485 et 487.
Dans un souci de clarté légitime, le classement formel et chronologique des œeuvres apparaît souvent, à tort ou à raison, comme un tout organique, et les critères stylistiques, comme le reflet d'une logique univoque, qu'une discussion sur les méthodes d'approche aurait permis de nuancer. Au-delà du constat de la rareté du schéma corinthien dans les exemples considérés comme précoces, l'auteur, par une étude de l'épannelage, aurait pu cerner l'impact résiduel de ce schéma sur la distribution du décor de palmettes et d'entrelacs, à l'instar de ses observations similaires sur les chapiteaux de Brive (fig. 1). Si l'on peut s'interroger sur la pertinence du classement des chapiteaux du " foyer très dynamique entre Vézère et Auvézère " d'après la nature et la position des éléments figuratifs sur la corbeille (p. 75-143), l'accent mis sur la forme crée une réelle disparité dans l'approche iconographique, où la notion de "programme " aurait d'ailleurs pu tenir compte des réflexions que d'autres auteurs, dont Marcello Angheben, ont plus amplement exposées et discutées. Quelques imprécisions ajoutent à l'impression mitigée de cette partie, un peu trop allusive pour satisfaire le lecteur captivé par l'abondance et la qualité artistique des chapiteaux historiés présentés.

La réimpression de la thèse de $M$. Angheben, Les chapiteaux romans de Bourgogne, aux éditions Brepols, rappelle l'intérêt de l'approche iconographique d'un corpus régional, composé de plus de cinq cents chapiteaux figurés et historiés. Face au débat sur la validité du concept même de "programme ", l'auteur entend entreprendre la recherche des " contours du champ sémantique" des thèmes dans le contexte d'une " syntaxe " spatiale décelée dans la répartition des chapiteaux (fig. 2), clef de lecture d'une interdépendance à condition d'écarter les coïncidences fortuites : " C'est donc au départ de séries plus ou moins importantes que les rapprochements de thèmes complémentaires ou antithétiques peuvent être considérés comme intentionnels" (p. 16). La démarche, érudite et stimulante, se heurte parfois à une vision partielle et partiale du cadre architectural : l'exposé inaugural sur les " thèmes spécifiques aux chœurs liturgiques " annonce d'emblée la difficulté d'en définir l'emprise (p. 21), et l'analyse des chapiteaux de Vézelay, pierre de touche de l'étude, inverse l'approche en proposant une relecture de la marche de la construction pour expliquer les perturbations du sens de lecture supposé (p. 427-434). La synthèse de l'étude des thèmes et de leur répartition spatiale met en relief l'Église comme espace symbolique, image du Paradis et de la Jérusalem

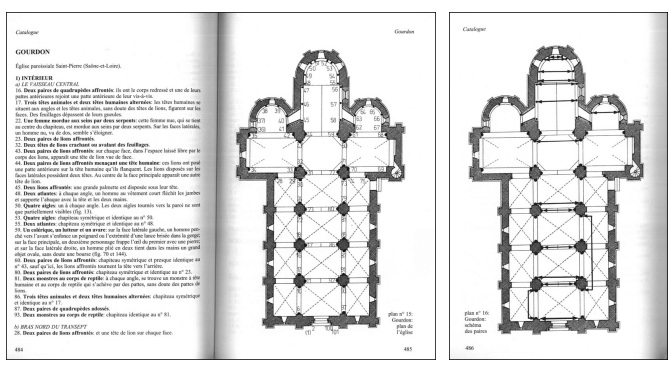

céleste, le combat de ses défenseurs et agresseurs, les paradigmes du combat spirituel, de la délivrance et du salut. Si le " clivage significatif " entre les forces du Bien et du Mal (fig. 3), fil conducteur $\mathrm{du}$ classement monographique des programmes (p. 403-447), tend à éclipser d'autres dénominateurs communs possibles, le commentaire des thèmes individuels, solidement documenté, offre une vision détaillée, nuancée et captivante des interprétations possibles comme de leur arrière-plan littéraire et exégétique.

La thèse de Caroline Roux, Les portails romans en Haute-Auvergne, inscrit l'approche du décor sculpté dans une étude plus vaste des rapports de l’ " architecture de passage ", celle des portes avec l'espace ecclésial et ses fonctions liturgiques, comme avec l'environnement de l'habitat et du cimetière, selon des modes et traditions qui influent sur la configuration et la disposition du ou des portails à l'ouest et/ou au sud de l'église. La rareté d'une sculpture d'envergure (tributaire surtout de la sculpture de l'ouest aquitain) appelle une réflexion originale et fructueuse sur un possible élargissement des perspectives de l'étude formelle, typologique, fonctionnelle et sémantique, qui sort des sentiers battus pour aborder des contextes rarement envisagés. Traditionnelle dans l'approche de la géographie stylistique, qui se fonde sur un inventaire publié dans un ouvrage à part ${ }^{2}$, la vision proposée innove par le croisement des axes d'enquête et par les questions qu'il soulève : entre autres, la simple notion de portail principal et secondaire, débattue depuis le colloque de 1990 sur la façade romane ${ }^{3}$, ne peut certes répondre à la diversité des cas et au désaccord possible entre la forme monumentale et la fonction, mais le réseau d'arguments avancés pour appréhender la position des portes, comme l'iconographie du décor, les conditions climatiques et les usages actuels, reste discutable (p. 194-202).

Lorsque É. Proust conclut à l'existence d'équipes de sculpteurs spécialisés dont la hiérarchie interne se distinguerait par une répartition des tâches lisibles dans la finition des œuvres (p. 203- 


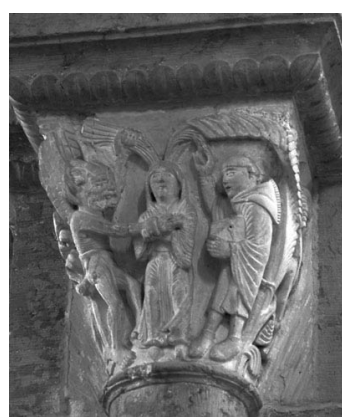

205), elle aborde une question importante qui aurait mérité l'apport d'une étude technique précise, pour mieux prendre ses distances à l'égard de la terminologie courante, inspirée des réalités plus tardives. Le concept $d^{\prime}$ " atelier ", de la distinction qualitative des intervenants et de l'itinérance des sculpteurs, sur laquelle se fonde l'interprétation traditionnelle des filiations artistiques, ne tient pas compte des critiques exprimées au cours des dernières décennies, certes dans des contextes géographiques et chronologiques différents ${ }^{4}$. Ainsi, l'existence de "corporations" de tailleurs de pierre strictu sensu reste à prouver. Et quelle trace palpable permettrait de restituer la " hiérarchie dans les aptitudes " et d'attribuer d'office aux " sculpteurs de deuxième catégorie ", désignés comme " compagnons " et apprentis " et privés de la réalisation des chapiteaux historiés selon la même hypothèse, la " lourdeur " d'exécution dont l'appréciation reste arbitraire?

Qu'il nous soit permis d'évoquer à cet égard nos propres travaux sur le portail ${ }^{5}$ et le cloître de Saint-Trophime d'Arles ${ }^{6}$ qui cherchent à démontrer l'utilité de l'analyse technique pour une meilleure compréhension des conditions et principes de la réalisation. Aux indices d'une préfabrication des éléments du décor du portail, tributaire de modèles graphiques, et de leur assemblage par une équipe autonome s'ajoute, pour le cloître, la répartition des tâches de la sculpture, de l'épannelage des blocs de marbre à la finition, selon des formes élémentaires qui distinguent d'emblée le décor végétal et historié. Or, la variation systématique des techniques de finition dans le détail trompe sur la rigueur des schémas figuratifs sous-jacents et interdit toute distinction claire de "styles " ou de " mains " individuels. L'exemple d'Arles pose ainsi la question fondamentale de la validité du concept traditionnel de l' "atelier " et des critères de la distinction des individus, oppose à l'hypothèse de l'itinérance des artistes celle de la circulation des modèles graphiques et souligne l'importance de l'étude du processus de fabrication, où les contraintes du matériau disponible, en particulier dans le cas du réemploi antique, peuvent jouer un rôle décisif.

Si l'étude technique du travail de la pierre peut donc apporter des éléments significatifs à l'étude formelle, son absence déçoit dans les travaux qui s'en réclament : ainsi, le terme d'épannelage, plusieurs fois évoqué par É. Proust, ne répond-il à aucune analyse concrète de la forme du bloc épannelé, dont l'importance n'est pourtant plus à démontrer depuis les recherches d'Éliane Vergnolle sur le clocher-porche de Saint-Benoît-surLoire $^{7}$ et celles de Dorothea Hochkirchen sur les chapiteaux inachevés des galeries hautes de Spire $^{8}$. Chez A. Autissier, une connaissance plus intime des techniques aurait permis d'éviter quelques erreurs, comme l'idée obsolète de l'extraction en carrière à l'aide de coins de bois humidifiés, et de placer la taille de la pierre au cœur du sujet.

Pour conclure, il nous paraît utile d'examiner la place du cadre régional, caractéristique de bien des thèses universitaires, dans la conception d'une exposition récente consacrée à la France romane dans son ensemble: La France romane au temps des premiers Capétiens (987-1152) ${ }^{9}$. Pour privilégier les critères artistiques, la partie principale de cette manifestation était organisée autour des "grands centres de la création ", selon une vision qui ne coïncide que partiellement avec le découpage politique du temps. Face à ce choix traditionnel et ses dangers inhérents, l'exposé de Jean-René Gaborit et Olivier Poisson (p. 210-211) souligne les échanges et autres facteurs qui créent des vecteurs d'unité dans la diversité, dont l' " osmose " entre les pouvoirs ecclésiastiques et laïcs, gage du rayonnement artistique des principaux centres de création qui dépasse souvent le simple clivage régional. De même, l'introduction générale de Jean-René Gaborit sur la sculpture architecturale, sensible au danger des visions stylistiques évolutionnistes et linéaires, insiste sur la complexité du phénomène et de sa relation avec les antécédents et modèles (p. 21-22). La nouveauté des décors extérieurs, le rapport de la sculpture avec les arts de la couleur et son rôle comme support de l'image rapprochent la sculpture monumentale des autres manifestations artistiques, une relation illustrée entre autres par la disposition des œuvres dans l'exposition. En revanche, si la synthèse finale sur les "grands thèmes du XII ${ }^{\mathrm{e}}$ siècle " accorde une place de choix à la fortune du chapiteau historié, elle a tendance à négliger le lien entre décor monumental et architecture, ce qui reflète l'approche muséale du sujet ${ }^{10}$.

1 Marie-Thérèse Camus, Claude Andrault-Schmitt éd., NotreDame-la-Grande de Poitiers. L'cuvre romane, Paris/Poitiers, 2002. 2 Caroline Roux, Portails romans de Haute-Auvergne. Études de sites, (Mémoires de la Société La Haute-Auvergne), Aurillac, 2004.
3. Vézelay, chapiteau avec la tentation de saint Benoît. 
3 Willibald Sauerländer, "Façade ou façades romanes?", dans La façade romane (colloque, Poitiers, 1990), Cahiers de civilisation médiévale, 34, fasc. 3-4, 1991, p. 393-401.

4 Dorothea Diemer, Untersuchungen zu Architektur und Skulptur des Abteikirche von Saint-Gilles, Stuttgart, 1978 ; Peter Kurmann, "Mobilité des artistes ou mobilité des modèles ? À propos de l'atelier des sculpteurs rémois ", dans Revue de l'Art, 120, 1998-2, p. 23-34 ; Andreas Hartmann-Virnich, " Sur les traces des sculpteurs et maçons. Les découvertes archéologiques ", dans A. Hartmann-Virnich éd., Le portail de SaintTrophimed'Arles. Naissance etrenaissance d'un chefd'xuvre roman, Arles, 1999, p. 107-157; A. Hartmann-Virnich, « Du programme décoratif à la mise en œuvre. Les chapiteaux du portail et de la galerie nord du cloître de Saint-Trophime d'Arles ", dans Apocalypse, visionsetreprésentations à l'époque romane. Recherches récentes sur l'art roman, (colloque, Issoire, 1998), Revue d'Auvergne, t. II, 116, 564-4, (2002) 2003, p. 33-71.

5 A. Hartmann-Virnich, "Sur les traces des sculpteurs et maçons. Les découvertes archéologiques ", dans HaRTMANNVIRNICH, 1999, cité n. 4, p. 107-157.

6 HaRTMANn-Virnich, 2002, cité n. 4, p. 33-71.

7 Eliane Vergnolle, Saint-Benoît-sur-Loire et la sculpture du $X I^{e}$ siècle, Paris, 1985.

8 Dorothea Hochkirchen, Mittelalterliche Steinbearbeitung und die unfertigen Kapitelle des Speyerer Domes, Cologne, 1990 ; Dorothea Hochkirchen, "Le travail de la pierre dans la cathédrale de Spire. Déductions sur l'entreprise et les phases de l'édification ", dans Chantiers médiévaux, Paris, 1996, p. 99-126.

9 La France romane au temps des premiers Capétiens (987-1152), Danielle Gaborit-Chopin éd., (cat. expo., Paris, musée du Louvre, 2005), Paris, 2005.

10 Sur le décor monumental, on se référera avec profit aux travaux des archéologues, qui nous en rappellent les « visages oubliés " : le musée Sainte-Croix de Poitiers a ainsi consacré une exposition au thème du stuc, dont l'image fragmentaire trompe sur l'importance de ce matériau qui étonne par la diversité des usages et la richesse des réalisations. Voir Christian Sapin éd., Le stuc. Visage oublié de l'art médiéval, (cat. expo., musée Sainte-Croix, Poitiers, 2004-2005), Paris/Poitiers, 2004

Andreas Hartmann-Virnich, Université de Provence, hartmann-virnich.andreas@neuf.fr

\section{L'architecture cistercienne, une forteresse historiographique}

\section{Claude Andrault-Schmitt}

- Peter Fergusson, Stuart Harrison, Rievaulx Abbey.

Community, Architecture, Memory, with contributions from
Glyn Coppack, New Haven/London, Yale University Press, 1999. 282 p., 189 fig., biblio. et index. ISBN : 0-300-07831-5; $84 € / 101 \$$.

- Thomas Coomans, L'abbaye de Villers-en-Brabant. Construction, configuration et signification d'une abbaye cistercienne gothique, Bruxelles, Racine et Brecht/Cîteaux, Commentarii cistercienses, (Studia et Documenta XI), 2000. 624 p., fig. n. et b. et coul., index. ISBN : 2-87386-200-9; $112 €$

- Megan CASsidy-Welch, Monastic Spaces and their Meanings. Thirteenth-Century English Cistercian Monasteries, (Medieval Church Studies, 1) Turnhout, Brepols, 2001. 293 p., 51 fig. n. et b., index. ISBN : 2-503-51089-2; $61 €$

- Terryl N. KINDER, Cistercian Europe. Architecture of Contemplation, Cistercian Publications, Kalamazoo, 2004. 407 p., fig. n. et b., pl. coul., index. ISBN : 0-8028-3887-1 $61,50 € / 73,85 \$$.

- Terryl N. KINDER éd., Perspectives for an Architecture of Solitude. Essays on Cistercians, Art and Architecture in Honour of Peter Fergusson, (Medieval Church Studies, 11), Turnhout, Brepols (Studia et Documenta, 13), Cîteaux/2004. 409 p., fig. n. et b. et coul., index. ISBN : 2-503-51692-0; $162 €$.

Bien plus que l'isolement supposé des sites ou la réalité des enceintes, l'Ordre était considéré par les cisterciens des $\mathrm{XII}^{\mathrm{e}}$ et $\mathrm{XIII}^{\mathrm{e}}$ siècles comme la "forteresse " les protégeant, car "ils savaient très bien que les limites entre monde monastique et monde non monastique nécessitaient d'être créées mentalement aussi bien que physiquement " (M. Cassidy-Welch). Reprenons cette métaphore pour nous demander si nous n'avons pas édifié une catégorie historiographique trop spécifique : il est significatif que beaucoup de titres utilisés ici sont issus de collections " cisterciennes". Pourtant, tous proposent une révision des idées reçues. Selon des articulations variées, de la monographie à la synthèse interdisciplinaire, ils tendent à relativiser l'identité cistercienne au sein du mouvement monastique, en s'éloignant d'une histoire que les moines blancs ont élaborée eux-mêmes ${ }^{1}$.

Les célébrations anniversaires de la naissance de Bernard de Clairvaux en 1990 et de la fondation de Cîteaux en 1998 ont entraîné colloques et publications ${ }^{2}$. Oublions ceux qui ne sacrifient qu'à l'esprit de commémoration, et notons pour les autres une réticence légitime face à l'analyse des formes architecturales, critiquée pour avoir été limitée aux abbatiales : aussi a-t-on assisté à une sorte de recentrage sur le patrimoine foncier et sa gestion, l'activité artisanale ou l'aménagement hydraulique. Les historiens de l'art se sont trouvés démunis face aux historiens, parmi lesquels les spécialistes de l'archéologie du paysage semblaient s'opposer aux grandes plumes exaltant les choix d'austérité. Le développement des études outreAtlantique, l'ouverture européenne, le rapport 